DOI: http://dx.doi.org/10.15359/rgac.1-56.5

Revista Geográfica de América Central. No 56

ISSN 1011-484X, enero-junio 2016

pp. 113-135

\title{
EL PENSAMIENTO GEOGRÁFICO EN LA PERCEPCIÓN DE RIESGOS POR PELIGROS HIDROMETEOROLÓGICOS EXTREMOS: ESTUDIO DE CASO MARIEL, CUBA
}

\author{
THE GEOGRAPHICAL THINKING IN THE PERCEPTION \\ OF RISKS FOR EXTREME HYDROMETEOROLOGICAL \\ HAZARDS: A CASE OF STUDY AT MARIEL, CUBA
}

\author{
Pablo Bayón Martínez ${ }^{1}$ \\ Instituto de Filosofía, CITMA, Cuba
}

\begin{abstract}
RESUMEN
Los fundamentos y avances geográficos en el análisis de la percepción de los riesgos ambientales, en tanto proceso construido social y culturalmente, ponen de manifiesto las profundas interconexiones entre los sistemas socioeconómico, político, ambiental y cultural, generados en una región o comunidad, articulando diversidad de unidades geoespaciales (ambientales). El artículo refiere estudio de caso que revela el significado del conocimiento geográfico de los entornos de vida cotidiana del sujeto (individual y colectivo), para identificación de vulnerabilidades sociogeográficas, en aras de la minimización de riesgos hidrometeorológicos extremos, lo que aporta a las metodologías de estudios (de percepción) en fase de elaboración y perfeccionamiento en Cuba.
\end{abstract}

Palabras clave: percepción de riesgo; riesgo de peligros naturales; vulnerabilidad social, formación cultural ambiental (geográfica).

1 Ms C. en Geografía, Medio Ambiente y Ordenamiento Territorial. Profesor e Investigador Auxiliar. Instituto de Filosofía, Ministerio de Ciencia, Tecnología y Medio Ambiente (CITMA). Calle Calzada No.251 esq. J, Vedado, La Habana. Cuba. CP-10400; Correo electrónico bayon@filosofia.cu pabamar@yahoo.com

Fecha de recepción: 06 de noviembre de 2015

Fecha de aceptación: 12 de enero de 2016 
Pablo Bayón Martinez. The geographical thinking in the perception of risks for extreme hydrometeorological hazards: a case of study at Mariel, Cuba

DOI: http://dx.doi.org/10.15359/rgac.1-56.5

\begin{abstract}
The geographic fundamentals and advances in the analysis of the perception of environmental risks, in socially and culturally developed processes, evidence the deep interconnections amongst socioeconomic, political, environmental and cultural systems generated in a region or community, thus articulating the diversity of the geospatial (environmental) units. This paper refers to a case study that reveals the meaning of geographical knowledge about the environment of daily life (individual and collective) in order to identify socio-geographic vulnerabilities in order to minimize extreme hydrometeorological hazards, which helps developing methodologies of study (perception) at the preparation and improvement stage in Cuba
\end{abstract}

Keyword: Perception of risks, risks of environmental hazards, social vulnerability, cultural and environmental formation (geographic)

\title{
Introducción
}

La emergencia de "la cuestión ambiental global", evidente desde la década de los 60's, del pasado siglo, constituye uno de los más abarcadores problemas del mundo contemporáneo y ha abierto nuevas perspectivas. De hecho, los problemas del medio ambiente no son solubles mediante los viejos paradigmas científicos tecnológicos de la modernidad.

La ocupación, apropiación y transformación del espacio geográfico, es un proceso cultural, porque se crean bienes materiales (en torno a las relaciones sociales), valores, modos de hacer, de pensar, de percibir el mundo, todo lo cual constituye el patrimonio cultural construido por la humanidad a lo largo de la historia.

La Geografia ${ }^{2}$ siempre ha considerado la complejidad de su "objeto" al tratar nada menos que toda una dimensión de la realidad, independientemente de las reducciones del campo de estudio del cual se trate. Por ello, ha desarrollado una amplia diversidad de líneas de pensamiento, que han respondido a visiones ideológico-filosóficas particulares: mecanicista, racionalista, sistémica, holista. Cada una de dichas corrientes ha visualizado lo ambiental desde una perspectiva particular, priorizando determinadas tradiciones geográficas, y elaborándose las categorías de análisis particulares a través de múltiples corrientes: determinismo, corológico-regional, posibilismo, teórico-cuantitativa, humanística, crítica, y ambiental (Lobato, R; 1995).

La reducción del riesgo de desastres, teniendo en cuenta que la vulnerabilidad como variable en el análisis del desastre es un reflejo de

2 La historia, concepción teórica, filosófica y/o científica de la Geografía, ha configurado extensos legajos impresos, y de oratoria, a lo largo de la historia humana. Una síntesis de "La Geografía como ciencias" se describe en el tabloide del Curso UPT: Geografía. 2002, (en línea) Disponible en: (www.medioambiente.cu) 
las condiciones físicas, sociales, económicas y ambientales, tanto individuales como colectivas, están configuradas por las actitudes, conductas e influencias socioeconómicas, políticas y culturales de que son objeto las personas, familias, comunidades y países.

El artículo aporta al análisis -dentro de la vulnerabilidad sociala la percepción del riesgo por peligros hidrometeorológicos extremos (fuertes vientos, inundaciones por intentas lluvias y/o penetraciones del mar) en Cuba, considerando los resultados del estudio de percepción realizado por el Grupo Nacional de Evaluación de Riesgo (GNER) de la Agencia de Medio Ambiente (AMA), al que se le incorpora la dimensión geográfica (relieve y paisajes), en la interpretación de los resultados de la entrevista aplicada 16626 individuos del país y de proceso de gestión-capacitación educativa, en temas relacionados con las ciencias geográficas (por demás ambiental), a actores-gestores locales del territorio correspondiente al estudio de caso.

Considerando los aportes del conocimiento geográfico en la construcción teórica y práctica del espacio, como concepción de medio ambiente, y para la necesaria formación cultural de los actores-gestores sociales, en el comportamiento espacial del ser humano, en los contextos respectivos; la situación problémica gira en torno a cuestionarnos ¿cómo el conocimiento geográfico (educación geográfica) contribuye a la percepción del riesgo por peligros hidrometeorológicos extremos, en los espacios/territorios de asimilación-reproducción social en el contexto del desarrollo local?

Desde esta perspectiva se expone y valida propuesta de concepción propositiva, de constatación empírica y actuación profesional, dirigida a revelar la contribución de la educación geográfica - a través de proceso de capacitación-intervención educativa- en la formación cultural ambiental para la percepción de riesgos y el desarrollo local, social sostenible.

\section{Desarrollo}

La sociedad mundial emergente, basada en el conocimiento, permite transformaciones tan profundas del sistema de la naturaleza, que los riesgos de destrucción "marchan al unísono con nuestra capacidad incrementada de creación de conocimientos" (...) (Delgado, C; 2005: 237), y por ende, de las acciones de transformación y asimilación de los espacios de vida. 
Pablo Bayón Martinez. The geographical thinking in the perception of risks for extreme hydrometeorological hazards: a case of study at Mariel, Cuba

DOI: http://dx.doi.org/10.15359/rgac.1-56.5

La Geografía es una ciencia que estudia al hombre en su interrelación dialéctica con la naturaleza (ciencia natural) y con otros hombres (ciencia social), la que ha incorporado la complejidad social y de su imaginario, mediante las percepciones diferentes del territorio, el paisaje y el ambiente, de vida social del sujeto. Opera con los conceptos geográficos fundamentales: espacio, paisaje, geosistema y región.

Como sistema de ciencias tiene que ver con todo lo que se difunde en la superficie de la Tierra, es decir, lo que se distingue por su espacialidad, lo que adquiere especial significado y relevancia para todas las actividades humanas, a partir de las propias prácticas sociales cotidianas, que generan y configuran y usan espacios de significación (práctica, de vida, simbólica, etc.).

La aproximación conceptual y metodológica de todos los enfoques desarrollados por la Geografía a lo largo de su historia, considerando a sus objetos como sistemas ambientales, espacio-temporales y territoriales que se forman en la superficie terrestre en el proceso de la interacción Naturaleza-Sociedad, condiciona la tendencia de acercar la noción de medio ambiente a la de espacio, con el propósito expreso de superar la dicotomía entre la Naturaleza y la Sociedad, y articular la cuestión ambiental a la creación de espacios.

El estudio social del riesgo y los desastres, asociados a las actividades humanas y/o a cualquier tipo de amenaza natural, han sido de gran importancia para los científicos sociales y no sólo de aquellos que se encargan del estudio del comportamiento humano, como los sicólogos o sociólogos, sino también, de otras disciplinas, como los geógrafos, antropólogos, historiadores etc.

La Geografía (de la percepción) y el estudio del subjetivismo han puesto de manifiesto que el "imaginario territorial" tiene gran importancia en el comportamiento espacial del ser humano; en la territorialidad donde existe cada "ser" (humano), configuradoras de espacios comunes, de relaciones múltiples dado por mediación de sus símbolos, sentidos y significados, reguladoras de sus prácticas sociales.

Mencionan Conesa y Calvo (2003) que el riesgo tiene una dimensión espacial porque se presenta en un territorio determinado, y es justo el análisis espacial, la clave del trabajo del geógrafo. La vocación de cartografiar los espacios y el tipo de riesgo que amenaza al "lugar", desde 
Pablo Bayón Martínez. El pensamiento geográfico en la percepción de riesgos por peligros hidrometeorológicos extremos: estudio de caso Mariel, Cuba

DOI: http://dx.doi.org/10.15359/rgac.1-56.5

la visión sistémica de los condicionantes naturales y sociales contextualmente, permite elaborar políticas de gestión basadas sobre todo dentro del concepto de "riesgo aceptable".

\section{Del estudio de percepción de peligros hidrometeorológicos ex- tremos en Cuba (2009-2011): concepción teórico-metodológica}

La metodología utilizada combina la intervinculación metodológica y teórica de las perspectivas de análisis provenientes de la Geografía, Filosofía de la Ciencia $^{3}$, de los estudios en Ciencia, Tecnología y Sociedad, así como de los estudios de percepción y comunicación del riesgo en los marcos del desarrollo local sostenible. En particular los estudios de peligro, vulnerabilidad y riesgo (PVR) del Grupo Nacional de Evaluación de Riesgos de la Agencia de Medio Ambiente, 2009-2011 (GNER-AMA; 2013).

El estudio realizado se inserta en una investigación descriptiva de corte interpretativo y desarrolla una metodología de análisis cualitativo con la utilización de elementos de la metodología cuantitativa. Desde el punto de vista metodológico se asume la estrategia de indagación y la base de datos, del grupo de expertos de percepción de o peligro, adscrita al Grupo Nacional de Evaluación de Riesgo de la Agencia de Medio Ambiente (op. cit) como parte de los estudios de peligro, vulnerabilidad y riesgo (PVR) por fuertes vientos, inundaciones por intensas lluvias y penetraciones del mar.

La aplicación de algunos métodos estadísticos multivariados ofrece una visión exploratoria de la percepción de los peligros de la población cubana.

La propuesta de investigación va dirigida a incluir la variable geográfica relativa a la posición topográfica del entorno físico, del ámbito de vida de los entrevistados: llanura, altura, montaña, paisaje; entorno costero, interior, fluvial, etc., lo que significa la inclusión de la perspectiva geográfica, en el análisis de la percepción de peligros naturales del sujeto de indagación, que aporte criterios para el diseño e implementación de una estrategia educativa, dirigida a la formación cultural ambiental ciudadana para la reducción del riesgo ante los peligros hidrometeorológicos que les

3 El tema de los peligros y desastres resulta oportuno si se toma en consideración la vocación de la Filosofía por el destino y la seguridad del hombre, con tal propósito resulta válido recordar la Tesis número 11 de Marx sobre Feuerbach: "Los filósofos no han hecho más que interpretar de diversos modos el mundo, pero de lo que se trata es de transformarlo". (Marx, 1974: 24-26) 
Pablo Bayón Martinez. The geographical thinking in the perception of risks for extreme hydrometeorological hazards: a case of study at Mariel, Cuba

DOI: http://dx.doi.org/10.15359/rgac.1-56.5

afecta, en sus modos de actuación cotidiano, articulándose con el desarro1lo sostenible local.

En tal sentido, se concibió el análisis de la dimensión geográfica inferida en el instrumento aplicado por la AMA, así como la propuesta de nuevas dimensiones y variables (del autor), complementándolos (tabla 1), las que han sido consideradas para la próxima actualización del estudio de percepción -a escala nacional- y que actualmente se aplica en la provincia La Habana (2015).

Tabla 1: Descripción operacional de dimensiones y variables aplicado por el estudio de percepción (GNER-AMA, 2009-11)

y propuestas de modificación del autor

\begin{tabular}{|c|c|c|}
\hline \multicolumn{3}{|c|}{$\begin{array}{l}\text { Definición operacional de la percepción del peligro por fuertes vientos e inundaciones por intensas } \\
\text { lluvias y/o penetraciones del mar. }\end{array}$} \\
\hline Dimensiones & \begin{tabular}{|c|} 
Variables \\
\end{tabular} & \begin{tabular}{|l|} 
Indicadores \\
\end{tabular} \\
\hline \multirow[t]{5}{*}{ Características sociodemográficas } & $\begin{array}{l}\text { - Sexo } \\
\text { - Edad }\end{array}$ & $\begin{array}{l}\text { masculino/femenino } \\
\text { joven/adulto/adulto mayor }\end{array}$ \\
\hline & - Nivel instrucción & $\begin{array}{l}\text { primaria/secundaria/medio } \\
\text { superior/superior }\end{array}$ \\
\hline & - Situación laboral & 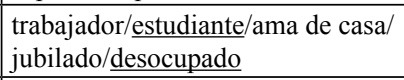 \\
\hline & - Ámbito de residencia & $\begin{array}{l}\text { urbano, rural, marginal }- \\
\text { comunidades eventuales/ } \\
\text { temporales }(i ?)\end{array}$ \\
\hline & $\begin{array}{l}\text { - Tiempo de residencia en el } \\
\text { barrio } \\
\text { - Condiciones de la vivienda }\end{array}$ & $\begin{array}{l}\text { menos de } 1 \text { año, entre } 1 \text { y tres } \\
\text { años, más de tres años } \\
\text { buenas/regulares/malas }\end{array}$ \\
\hline Principales actores & - identificación actores claves & $\begin{array}{l}\text { gobierno } \\
\text { empresa } \\
\text { otras instituciones } \\
\text { comunidad } \\
\end{array}$ \\
\hline \multirow[t]{2}{*}{$\begin{array}{l}\text { Actividad de manejo de los } \\
\text { peligros }\end{array}$} & $\begin{array}{l}\text { - Influencia del peligro en la } \\
\text { actividad económica }\end{array}$ & alto/medio/bajo \\
\hline & - Estrategias de manejo & $\begin{array}{l}\text { políticas de protección ambiental } \\
\text { comunicación y divulgación de } \\
\text { peligro } \\
\text { grado de preparación del sujeto } \\
\text { (trabajador, escolar, ciudadano). } \\
\underline{\text { Influencia del entorno institucional }} \\
\begin{array}{l}\text { y comunitario en la formación } \\
\text { cultural ambiental (EA). }\end{array}\end{array}$ \\
\hline
\end{tabular}


Pablo Bayón Martínez. El pensamiento geográfico en la percepción de riesgos por peligros hidrometeorológicos extremos: estudio de caso Mariel, Cuba

DOI: http://dx.doi.org/10.15359/rgac.1-56.5

\begin{tabular}{|c|c|c|}
\hline \multicolumn{3}{|c|}{$\begin{array}{c}\text { Definición operacional de la percepción del peligro por fuertes vientos e inundaciones por intensas } \\
\text { lluvias y/o penetraciones del mar. }\end{array}$} \\
\hline Dimensiones & Variables & Indicadores \\
\hline \multirow{6}{*}{$\begin{array}{l}\text { Rasgos de las percepciones de } \\
\text { peligro. }\end{array}$} & - Conocimiento de causas & antrópicas/naturales/otras \\
\hline & $\begin{array}{l}\text { - conocimiento de las } \\
\text { afectaciones }\end{array}$ & $\begin{array}{l}\text { afectaciones identificadas } \\
\text { grado de peligrosidad, severidad } \\
\text { conocimiento afectaciones } \\
\text { salud humana a la economía, al } \\
\text { territorio, grupos poblacionales. }\end{array}$ \\
\hline & $\begin{array}{l}\text { conocimiento de las } \\
\text { vulnerabilidades ante afectaciones. }\end{array}$ & $\begin{array}{l}\text { grado de vulnerabilidad -física- } \\
\text { ante el peligro y sus afectaciones }\end{array}$ \\
\hline & $\begin{array}{l}\text { - caracterización de las } \\
\text { afectaciones y evaluación de las } \\
\text { afectaciones }\end{array}$ & $\begin{array}{l}\text { tiempo de recuperación de las } \\
\text { afectaciones } \\
\text { afectaciones (identificadas) } \\
\text { identificación de daños } \\
\text { condiciones para la protección } \\
\text { identificación de acciones de } \\
\underline{\text { las personas para disminuir o }} \\
\underline{\text { aumentar las afectaciones }} \\
\underline{\text { Identificación de actores (sociales) }} \\
\underline{\text { Sentimientos ante las afectaciones. }}\end{array}$ \\
\hline & - Valoración del riesgo & alto/medio /bajo \\
\hline & - Propuestas de soluciones & $\begin{array}{l}\text { Sugerencias, identificación } \\
\text { de acciones de las personas } \\
\text { para disminuir o aumentar las } \\
\text { afectaciones }\end{array}$ \\
\hline \multirow[t]{5}{*}{ Geográficas } & - $\frac{\text { Relieve (posición topográfica }}{\underline{\text { del asentamiento }}}$ & $\begin{array}{l}\text { Llanura; } \\
\text { altura, } \\
\text { Montaña } \\
\text { Cuenca fluvial (curso superior, } \\
\text { medio, bajo) } \\
\text { Zona costera }\end{array}$ \\
\hline & Ámbito comunidad & $\begin{array}{l}\text { Urbana } \\
\text { Rural }\end{array}$ \\
\hline & $\begin{array}{l}\text { Magnitud; Frecuencia del } \\
\text { fenómeno }\end{array}$ & $\begin{array}{l}\text { Por impacto del fenómeno } \\
\text { peligroso } \\
\text { Por afectaciones: inundación } \\
\end{array}$ \\
\hline & $\begin{array}{l}\text { Conocimiento de la vulnerabilidad } \\
\text { (de ámbito físico-natural) }\end{array}$ & $\begin{array}{l}\text { Por condiciones geográficas: } \\
\text { relieve, zona costera, cuenca } \\
\text { fluvial } \\
\text { Estado/vivienda (buena, regular, } \\
\text { mala) }\end{array}$ \\
\hline & $\begin{array}{l}\text { Condiciones para enfrentar el } \\
\text { fenómeno }\end{array}$ & $\begin{array}{l}\text { Acciones/actores para reducir } \\
\text { riesgo ante el peligro contextual: } \\
\text { plan sujeto social (individual y/o } \\
\text { colectivo); alimentos; salud, y otro. }\end{array}$ \\
\hline
\end{tabular}

Fuente: (Modificado/autor. En "rojo y/o subrayado", las precisiones o sugerencias al comité de expertos del grupo de PVR-AMA para el estudio de percepción -en ejecución- en La Habana (2015), en el que se incluyen elementos de vulnerabilidad del entorno físico del sujeto de indagación. 
Pablo Bayón Martinez. The geographical thinking in the perception of risks for extreme hydrometeorological hazards: a case of study at Mariel, Cuba

DOI: http://dx.doi.org/10.15359/rgac.1-56.5

Los datos fueron procesados a través del programa computarizado (estadístico) IBM SPSS v.22 (Statistic Package Social Sciences), lo que posibilitó el análisis de correspondencias múltiples y simples, así como explorar las características de los individuos; así como para el examen de la influencia de algunas variables sobre la percepción de peligro, articulado con los objetivos de la investigación. Las variables se cruzaron con indicadores sociodemográficos (sexo, edad, nivel de instrucción, ocupación, tiempo de residencia en el consejo, tamaño del núcleo familiar, etc.) obteniéndose tablas de contingencia y de frecuencia, así como tablas de respuestas múltiples, articulando los resultados de percepción de peligros (alto-medio-bajo) obtenidos con los rasgos del complejo de relieve (llanura-altura-montaña) y paisaje geográfico predominante por cada unidad territorial (país, región, provincia, municipio, consejo popular).

\section{Percepción de riesgo por peligros naturales en el municipio Ma- riel: generalidades del contexto provincial}

El Municipio Mariel se ubica en la Región Occidental de Cuba, enmarcada en la nueva provincia de Artemisa ${ }^{4}$, en su porción litoral norte-centro. Como contexto político administrativo general, los datos generales sociodemográficos de la provincia (ONEI, 2010) que se describen en la (figura 1).

4 La Asamblea Nacional del Poder Popular aprobó el 1 de agosto de 2010 la Ley Modificativa de la Ley No. 1304 de 3 julio de 1976 "Ley de la División Político Administrativa", y con ello la Isla cuenta ahora con dos nuevas provincias, a partir del territorio de la actual provincia de La Habana. Ellas son: Artemisa y Mayabeque. La actual provincia de Ciudad de La Habana recupera su nombre histórico de provincia La Habana. Se reorganizan municipios en las provincias de Matanzas y Guantánamo 
Figura 1. Localización de la provincia Artemisa y sus municipios

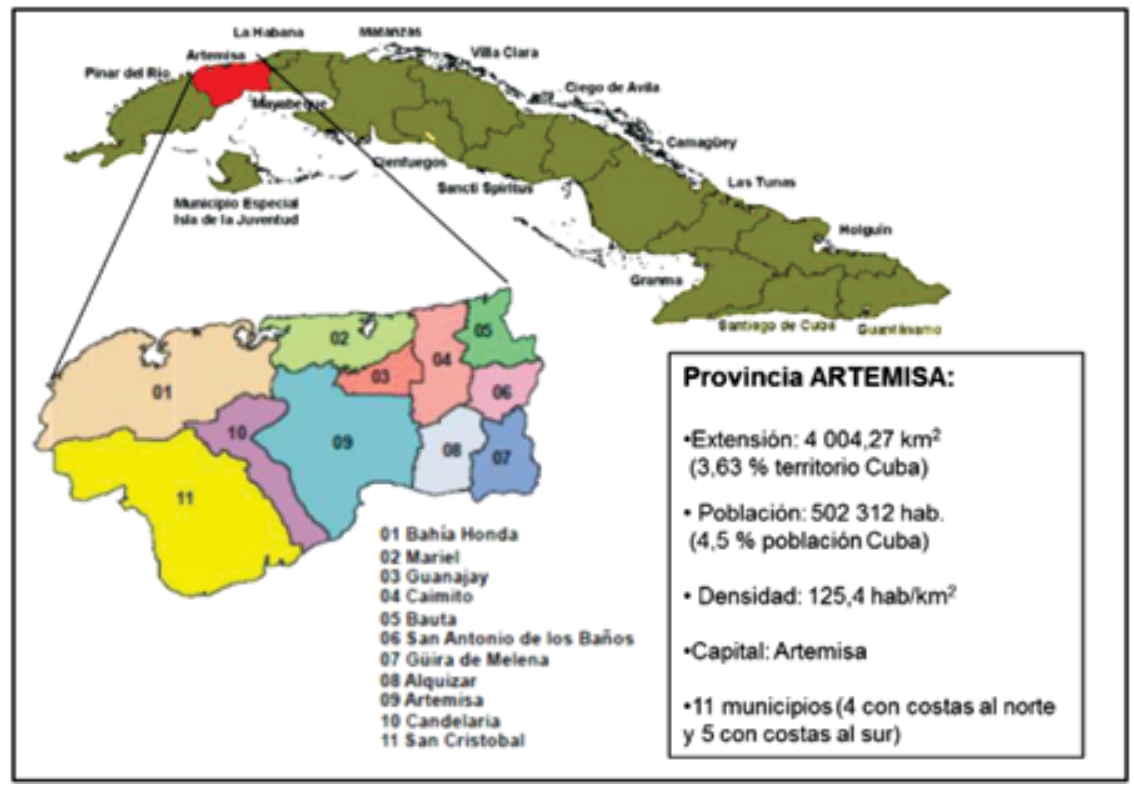

Fuente: ONEI, 2010.

En la figura 2, como referente general, se describen los resultados de percepción de peligros hidrometeorológicos por provincias. Puntualmente, las provincias más occidentales (Pinar del Río, Artemisa, Mayabeque e Isla de la Juventud) tienen los mejores índices de percepción de peligros (excepto Matanzas), aunque difieren en cuanto al tipo. Llama la atención el significativo predominio de percepción media-baja (que supera el 50\% del total de los encuestados), de sujetos que tienen una insuficiente o errónea percepción de los peligros referidos y las maneras de enfrentarlos, en las provincias Matanzas, Villa Clara, Holguín, Granma, Santiago de Cuba y Guantánamo, lo que han de ser tenidos en cuenta, en el diseño de acciones de educación y comunicación para la formación ambiental en los modos de actuación social contextualmente. 
Pablo Bayón Martinez. The geographical thinking in the perception of risks for extreme hydrometeorological hazards: a case of study at Mariel, Cuba

DOI: http://dx.doi.org/10.15359/rgac.1-56.5

Figura 2

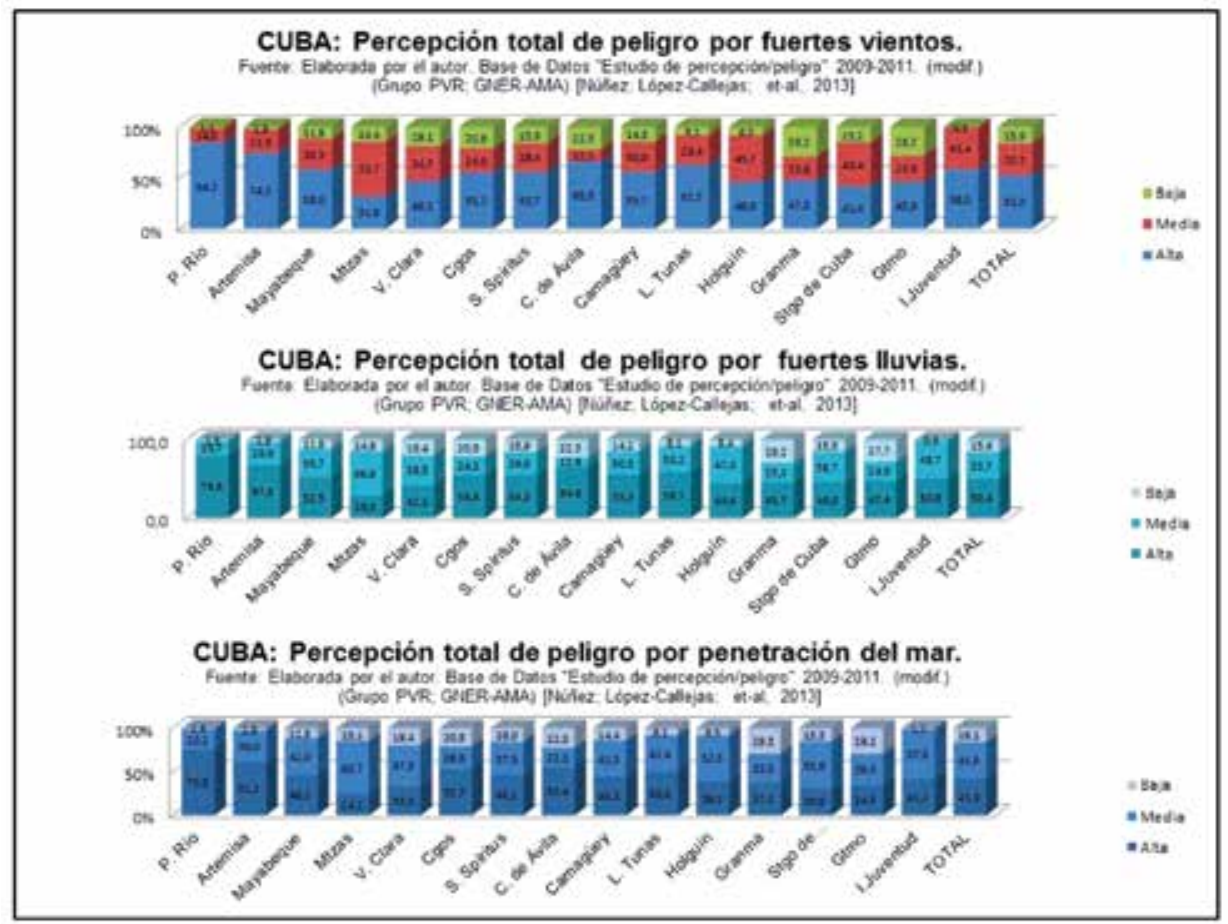

A escala nacional, el 85,8 \% de los encuestados reconocen al peligro de inundaciones por lluvias como el que más los afecta, el 85,3\% distingue al viento, y por último, solo el 18,6\%, a las penetraciones del mar.

En la provincia Artemisa y el municipio Mariel existe predominio de percepción "alta" de los peligros estudiados, con destaque para el peligro asociado con intensos vientos como máxima prioridad, que resulta directamente proporcional con la historia de ocurrencia de fenómenos meteorológicos extremos hacia esta área del país, con más de 80 impactos de huracanes entre los años 1800-2012 de acuerdo con datos del Anuario Estadístico de Cuba (ONEI,2012: 49), que representa el 44,0\% de estos eventos en todo el archipiélago. Otro hecho significativo es que durante los 10 años de precedencia al estudio de percepción referido, en el territorio han ocurrido más de 15 huracanes (más del 60\%), lo que coadyuva a la memoria histórica de los lugareños. 
Pablo Bayón Martínez. El pensamiento geográfico en la percepción de riesgos por peligros hidrometeorológicos extremos: estudio de caso Mariel, Cuba

DOI: http://dx.doi.org/10.15359/rgac.1-56.5

En la tabla 2, se describe el grado de percepción de peligro según unidad territorial de comparación en torno al estudio de caso. Son significativos los valores "medios" y "bajos" de los sujetos indagados, lo que a criterio del autor del presente estudio, representan un número significativo de población que requiere atención educativa para percibir y minimizar vulnerabilidades a escala local.

Tabla 2. Descripción del grado de percepción de peligros hidrometeorológicos que aportó la encuesta nacional

\begin{tabular}{|c|c|c|c|c|c|c|c|c|c|}
\hline \multirow{3}{*}{ Unidad territorial } & \multicolumn{9}{|c|}{ Nivel de percepción de peligros hidrometeorológicos (\%) } \\
\hline & \multicolumn{3}{|c|}{ VIENTO } & \multicolumn{3}{|c|}{ LLUVIA } & \multicolumn{3}{|c|}{ MAR } \\
\hline & Alta & Medio & Baja & Alta & Medio & Baja & Alta & Medio & Baja \\
\hline Región Occidental & 56,1 & 36,0 & 7,9 & 51,3 & 40,7 & 8,0 & 45,8 & 45,9 & 8,3 \\
\hline Provincia Artemisa & 74,3 & 22,9 & 2,8 & 67,3 & 29,9 & 2,8 & 61,2 & 36,0 & 2,8 \\
\hline Municipio Mariel & 54,5 & 36,4 & 9,1 & 47,7 & 43,2 & 9,1 & 50,0 & 40,9 & 9,1 \\
\hline
\end{tabular}

Fuente: Elaborada por el autor. Base de Datos "Estudio de percepción/peligro" 2009-2011. (modif.) (Grupo PVR; GNER-AMA) [Núñez, López-Callejas; et al; 2013]

Los estudios realizados por la Delegaciones CITMA $^{5}$ de la provincia Artemisa y del Municipio Mariel (CAP, 2012), descritos en la figura 3, clasifican el riesgo "alto" para cualquier intensidad de peligro por fuertes vientos, para todo el Municipio, ubicado entre los de mayor vulnerabilidad $\operatorname{total}^{6}(\mathrm{Vt})$ en la provincia (junto con Alquízar y Artemisa). Las áreas de peligro de inundación por penetración del mar afectan entre el 0,92-15,8 $\mathrm{Km}^{2}$ del municipio, lo que representa el 5,8\% del mismo, perteneciente a los CP: Boca-Mujica-Henequén, Mariel, Quiebra Hacha y Cabañas. Los asentamientos mayormente expuestos son: Henequén Viejo, La Boca, Vista del Mar y Granma.

5 CITMA: Ministerio de Ciencia, Tecnología y Medio Ambiente. Cuba.

6 Resulta de la sumatoria de todas las vulnerabilidades (estructural, no estructural, funcional, social, ecológica, económica) del Consejo Popular o de cualquier unidad político administrativa ante un determinado peligro (GNER-AMA, 2010: 7) 
Pablo Bayón Martínez. The geographical thinking in the perception of risks for extreme hydrometeorological hazards: a case of study at Mariel, Cuba

DOI: http://dx.doi.org/10.15359/rgac.1-56.5

Figura 3. Provincia Artemisa; Cuba: resumen de riesgos según escenario de peligro. (Municipio Mariel, estudio de caso)

(Marcado en "línea de punto”, el área aproximada del Municipio Mariel)

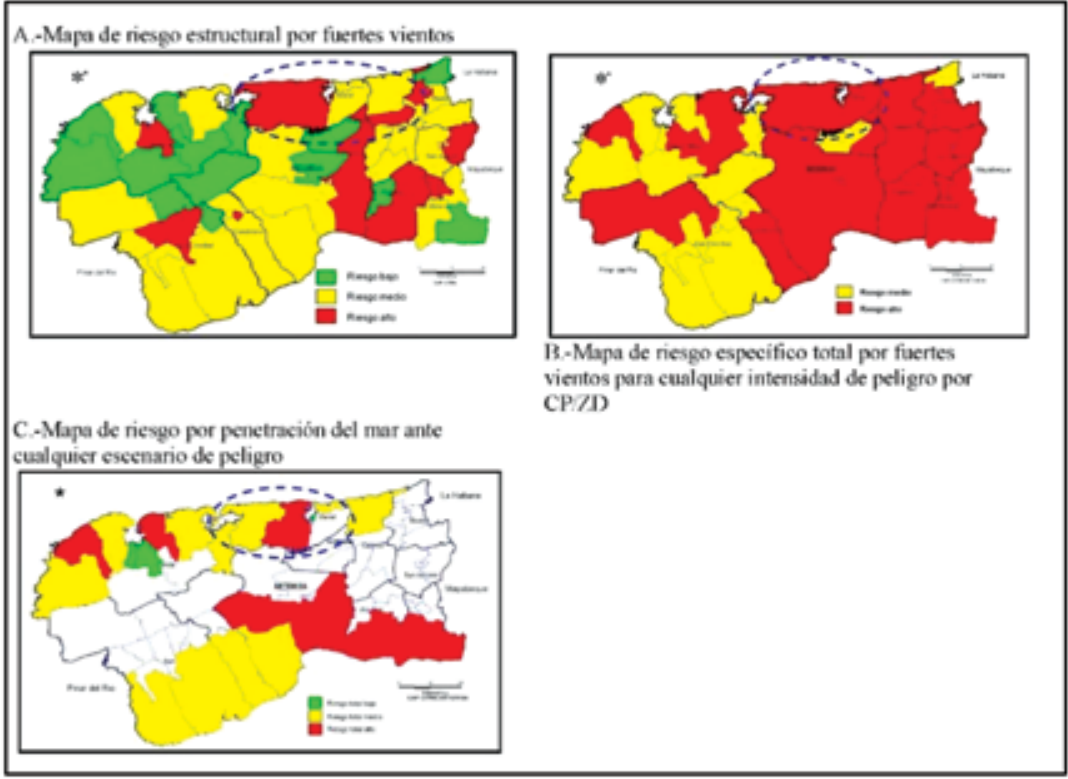

Fuente: Consejo de la Administración Provincial, Artemisa. (2012). (simplificado).

\section{Práctica de gestión-intervención educativa en territorio estudio de caso}

Con el propósito de valorar la contribución del conocimiento geográfico a la formación cultural del sujeto en la percepción de los peligros hidrometeorológicos estudiados, articulando con procesos de gestión de conocimientos desarrolladas internacionalmente y en Cuba, y dado el carácter multidisciplinario de estos estudios, la investigación marco se insertó en el proyecto de gestión del conocimiento del Departamento de Extensión Universitaria del Instituto Superior Politécnico "José Antonio Echevarría" (ISPJAE), en el posgrado "Conocimientos elementales para el tratamiento de estudios ambientales locales", como contribución a la capacitación de gestores-decisores comunitarios del Municipio Mariel, circunstancia que coadyuvó a la constatación empírica del estado de percepción inicial y final del grado de conocimientos ante los peligros -objeto 
de estudio- y de sus vulnerabilidades (sociales, individuales, geográficas y otras) respectiva, por parte del sujeto (cursista).

Como parte del proceso de capacitación, se concibió encuesta aplicada a los participantes del curso, como técnica del paradigma cuantitativo, articulando con la metódica de indagación cualitativa, dado el carácter de la "percepción de riesgos por peligros hidrometeorológicos extremos" por los individuos, que permite su aplicación en el estudio de un mismo fenómeno como parte de la triangulación metodológica.

Estos atributos - a criterio del autor- constituyen referentes generales que modelan la concepción de acciones educativas, de formación cultural general de capacidades y habilidades cognitivas, que redunden en la percepción de los peligros y pauten los modos de actuación individual y colectiva del sujeto en su accionar cotidiano (Bayón, P.; 2014).

Se aplica y enriquece contextualmente las metodologías de estudios aplicados por Puy (1994: 239), que retoma la propuesta del grupo de Oregón (Fischoff et al.; 1978; Slovic et al., 1980, 1985), citado por Puy (1994: 240); que incluye nueve atributos de valoración de las percepciones de riesgos; que retoma Almaguer (2008: 59) en localidades del oriente cubano. En nuestro ejercicio, se incluyen doce atributos que contemplan -además- los factores de aceptabilidad de riesgo descritas por Velk y Stallen (1980, citado por Espluga, 2002), se describe en la tabla 3. 
Pablo Bayón Martínez. The geographical thinking in the perception of risks for extreme hydrometeorological hazards: a case of study at Mariel, Cuba

DOI: http://dx.doi.org/10.15359/rgac.1-56.5

Tabla 3: Escalas de atributos cualitativos de percepción de peligros hidrometeorológicos, para valorar la actitud del sujeto según escala de tipo Likert, concebidos a encuesta de percepción de peligro hidrometeorológicos aplicada en curso de gestión de conocimiento en Mariel

\begin{tabular}{|c|c|c|c|c|c|c|c|c|}
\hline \multirow[t]{3}{*}{ INVOLUNTARIO } & \multicolumn{7}{|c|}{ Voluntariedad del riesgo ante el peligro } & \multirow[t]{3}{*}{ VOLUNTARIO } \\
\hline & \multicolumn{7}{|c|}{$\begin{array}{l}\text { II- } 1 \text { ¿Se enfrenta usted a este peligro de forma voluntaria o } \\
\text { involuntaria? }\end{array}$} & \\
\hline & $\begin{array}{l}\text { Riesgo } \\
\text { INVOLUNTARIO }\end{array}$ & 1 & 2 & 3 & 4 & 5 & $\begin{array}{l}\text { Riesgo } \\
\text { VOLUNTARIO }\end{array}$ & \\
\hline \multirow[t]{3}{*}{ INMEDIATO } & \multicolumn{7}{|l|}{ Inmediatez del efecto } & \multirow[t]{3}{*}{ RETARDADO } \\
\hline & \multicolumn{7}{|c|}{$\begin{array}{l}\text { II-2 En caso de ocurrir ¿cuándo se experimentarían los efectos } \\
\text { más nocivos de este peligro? }\end{array}$} & \\
\hline & $\begin{array}{l}\text { Efecto } \\
\text { INMEDIATO }\end{array}$ & 1 & 2 & 3 & 4 & 5 & \begin{tabular}{|l} 
Efecto \\
RETARDADO \\
\end{tabular} & \\
\hline \multirow{3}{*}{$\begin{array}{l}\text { DESCONOCIDO } \\
\text { (cognitivo/ } \\
\text { vulnerable) }\end{array}$} & \multicolumn{7}{|c|}{ Conocimiento del riesgo ante el peligro } & \multirow{3}{*}{$\begin{array}{l}\text { CONOCIDO } \\
\text { (cognitivo/no } \\
\text { vulnerable) }\end{array}$} \\
\hline & \multicolumn{7}{|c|}{$\begin{array}{l}\text { II-3 ¿En qué medida usted conoce el riesgo asociado a este } \\
\text { peligro? (daños que puede causarle, posibilidades que tiene de } \\
\text { experimentar estos daños, etc.) }\end{array}$} & \\
\hline & $\begin{array}{l}\text { Nivel de riesgo } \\
\text { DESCONOCIDO }\end{array}$ & 1 & 2 & 3 & 4 & 5 & $\begin{array}{l}\text { Nivel de riesgo } \\
\text { CONOCIDO con } \\
\text { precisión }\end{array}$ & \\
\hline \multirow{3}{*}{$\begin{array}{l}\text { DESCONOCIDO } \\
\text { (líderes- } \\
\text { comunidad/ } \\
\text { responsables) }\end{array}$} & \multicolumn{7}{|c|}{$\begin{array}{l}\text { Conocimiento del riesgo (ciencia; instituciones; gestores } \\
\text { comunitarios) }\end{array}$} & \multirow{3}{*}{$\begin{array}{l}\text { CONOCIDO } \\
\text { (líderes-comunidad } \\
\text { responsables) }\end{array}$} \\
\hline & \multicolumn{7}{|c|}{$\begin{array}{l}\text { II-4 ¿En qué medida conoce la ciencia y los responsables de la } \\
\text { comunidad a este peligro? }\end{array}$} & \\
\hline & $\begin{array}{l}\text { Nivel de riesgo } \\
\text { DESCONOCIDO }\end{array}$ & 1 & 2 & 3 & 4 & 5 & $\begin{array}{l}\text { Nivel de riesgo } \\
\text { CONOCIDO con } \\
\text { precisión }\end{array}$ & \\
\hline \multirow{3}{*}{$\begin{array}{l}\text { NO } \\
\text { CONTROLABLE } \\
\text { (percepción/ } \\
\text { vulnerable) }\end{array}$} & \multicolumn{7}{|c|}{ Control (personal) sobre el riesgo con relación al peligro } & \multirow{3}{*}{$\begin{array}{l}\text { CONTROLABLE } \\
\text { (percepción/ } \\
\text { no-vulnerable) }\end{array}$} \\
\hline & \multicolumn{7}{|c|}{$\begin{array}{l}\text { II-5 ¿Hasta qué punto usted puede, por habilidad personal o } \\
\text { diligencia, intervenir para controlar el daño que puede causarle } \\
\text { este peligro? }\end{array}$} & \\
\hline & $\begin{array}{l}\text { El riesgo personal NO SE } \\
\text { PUEDE CONTROLAR }\end{array}$ & 1 & 2 & 3 & 4 & 5 & $\begin{array}{l}\text { El riesgo personal } \\
\text { SE PUEDE } \\
\text { CONTROLAR } \\
\end{array}$ & \\
\hline \multirow[t]{3}{*}{ NUEVO } & \multicolumn{7}{|c|}{ Novedad del riesgo relacionado con el peligro } & \multirow[t]{3}{*}{ ANTIGUO } \\
\hline & \multicolumn{7}{|c|}{$\begin{array}{l}\text { II-6 En términos de nuevo-novedoso o antiguo-familiar, este } \\
\text { peligro es para usted o su comunidad. }\end{array}$} & \\
\hline & NUEVO & 1 & 2 & 3 & 4 & 5 & ANTIGUO & \\
\hline
\end{tabular}


Pablo Bayón Martínez. El pensamiento geográfico en la percepción de riesgos por peligros hidrometeorológicos extremos: estudio de caso Mariel, Cuba

DOI: http://dx.doi.org/10.15359/rgac.1-56.5

\begin{tabular}{|c|c|c|c|c|c|c|c|c|}
\hline \multirow[t]{3}{*}{$\begin{array}{l}\text { NO } \\
\text { CATASTRÓFICO }\end{array}$} & \multicolumn{7}{|c|}{$\begin{array}{l}\text { Constante/individual-Catastrófico el riesgo que provoca el } \\
\text { peligro }\end{array}$} & \multirow[t]{3}{*}{ CATASTRÓFICO } \\
\hline & \multicolumn{7}{|c|}{$\begin{array}{l}\text { II-7 ¿En qué grado este peligro que puede dañar a un gran } \\
\text { número de personas de una sola vez? }\end{array}$} & \\
\hline & CONSTANTE/individual & 1 & 2 & 3 & 4 & 5 & CATASTRÓFICO & \\
\hline \multirow[t]{3}{*}{ NO TEMIDO } & \multicolumn{7}{|c|}{ No temido-temido } & \multirow[t]{3}{*}{ TEMIDO } \\
\hline & \multicolumn{7}{|c|}{$\begin{array}{l}\text { II- } 8 \text { ¿En qué grado usted ha aprendido a convivir con dicho } \\
\text { peligro y no teme, o es uno al que le tiene gran temor? }\end{array}$} & \\
\hline & NO TEMIDO & 1 & 2 & 3 & 4 & 5 & TEMIDO & \\
\hline \multirow[t]{3}{*}{$\begin{array}{l}\text { DESCONOCIDO } \\
\text { (entorno) }\end{array}$} & \multicolumn{7}{|c|}{$\begin{array}{l}\text { Conocimiento de vulnerabilidad del entorno de vida - } \\
\text { individual y colectiva- ante el riesgo del peligro }\end{array}$} & \multirow[t]{3}{*}{$\begin{array}{l}\text { CONOCIDO } \\
\text { (entorno) }\end{array}$} \\
\hline & \multicolumn{7}{|c|}{$\begin{array}{l}\text { II-9 ¿En qué medida usted conoce la exposición al riesgo } \\
\text { asociado al peligro en cuestión, según las características del } \\
\text { entorno de vida cotidiana? }\end{array}$} & \\
\hline & $\begin{array}{l}\text { Nivel de vulnerabilidad } \\
\text { DESCONOCIDO }\end{array}$ & 1 & 2 & 3 & 4 & 5 & $\begin{array}{l}\text { Nivel de } \\
\text { vulnerabilidad } \\
\text { CONOCIDO }\end{array}$ & \\
\hline \multirow{3}{*}{$\begin{array}{l}\text { DESCONOCIDO } \\
\text { (vulnerabilidad/ } \\
\text { sujeto) }\end{array}$} & \multicolumn{7}{|c|}{$\begin{array}{l}\text { Conocimiento de vulnerabilidad de sufrir daño personal de } \\
\text { producirse el evento peligroso }\end{array}$} & \multirow{3}{*}{$\begin{array}{l}\text { CONOCIDO } \\
\text { (vulnerabilidad/ } \\
\text { sujeto) }\end{array}$} \\
\hline & \multicolumn{7}{|c|}{$\begin{array}{l}\text { II-10. La posibilidad de que Ud. experimente un daño como } \\
\text { consecuencia de este peligro es: }\end{array}$} & \\
\hline & $\begin{array}{l}\text { Nivel de vulnerabilidad } \\
\text { DESCONOCIDO }\end{array}$ & 1 & 2 & 3 & 4 & 5 & $\begin{array}{l}\text { Nivel de } \\
\text { vulnerabilidad } \\
\text { CONOCIDO }\end{array}$ & \\
\hline \multirow[t]{3}{*}{ POCO GRAVE } & \multicolumn{7}{|c|}{ Gravedad de las consecuencias } & \multirow[t]{3}{*}{ MUY GRAVE } \\
\hline & \multicolumn{7}{|c|}{$\begin{array}{l}\text { II- } 11 \text { En caso de producirse, la gravedad del daño que le puede } \\
\text { causar este peligro es: }\end{array}$} & \\
\hline & $\begin{array}{l}\text { Seguro que no va a ser } \\
\text { MORTAL }\end{array}$ & 1 & 2 & 3 & 4 & 5 & $\begin{array}{l}\text { Seguro que va a } \\
\text { ser MORTAL }\end{array}$ & \\
\hline & \multicolumn{7}{|c|}{ Autogestión de riesgo del peligro } & \\
\hline \multirow[t]{3}{*}{$\begin{array}{l}\text { NO } \\
\text { CONTROLABLE } \\
\text { (baja-percepción) }\end{array}$} & \multicolumn{7}{|c|}{$\begin{array}{l}\text { II-12. ¿En qué grado usted puede evitar que este peligro } \\
\text { desencadene una situación de consecuencias de negativas } \\
\text { (gravedad) para su vida y recursos personales? }\end{array}$} & CONTROLABLE \\
\hline & $\begin{array}{l}\text { Nivel de vulnerabilidad } \\
\text { DESCONOCIDO }\end{array}$ & \begin{tabular}{|l|}
1 \\
\end{tabular} & 2 & 3 & 4 & 5 & $\begin{array}{l}\text { Nivel de } \\
\text { vulnerabilidad } \\
\text { CONOCIDO } \\
\end{array}$ & \\
\hline & & & & & & & & \\
\hline
\end{tabular}

Fuente: Elaborado por el autor, adaptado de Slovic et al. (1995), citado por Puy (1994)

Se incluyen las características socio-demográficas generales de los individuos entrevistados (edad, sexo, nivel de escolaridad y ocupación), pues en varios estudios se ha comprobado que existe cierta relación entre 
Pablo Bayón Martinez. The geographical thinking in the perception of risks for extreme hydrometeorological hazards: a case of study at Mariel, Cuba

DOI: http://dx.doi.org/10.15359/rgac.1-56.5

estas y las percepciones de peligros y riesgos según la literatura consultada, que matizan la actitud o disposición sicológica de las personas a través de la propia experiencia (Slovic y Weber, 2002; Puy, 1994; Puy y Aragonés, 2003; Castañeda, 2014).

Con la finalidad de medir las diferentes variables o atributos del riesgo en los sujetos indagados (cursistas que constituyen habitantes expuestos), se empleó el enfoque psicométrico antes mencionado, empleando la combinación de Escala del tipo Likert de 5 puntos con un diferencial semántico, otorgándose un punto como puntuación mínima al ítem y 5 puntos a una respuesta que otorga el valor máximo al ítem propuesto. Esta medición indirecta se realiza por medio de unas escalas (Tabla 3), en las que partiendo de una serie de afirmaciones, proposiciones o juicios, sobre los que los individuos manifiestan su opinión, se deducen o infieren las actitudes, que son inferidas de las expresiones verbales o de la conducta observad en el sujeto.

El análisis de los datos permite la creación del perfil característico de la percepción para cada tipo de peligro, la construcción gráfica de los diferentes perfiles ofrece una panorámica descriptiva de las valoraciones realizadas por los asistentes al curso, que pueda ser contrastada con los resultados finales del mismo, como experiencia formativa (figura 4 A y B).

Figura 4. Perfil del riesgo percibido para los peligros hidrometeorológicos externos (Según atributos y riesgo global)

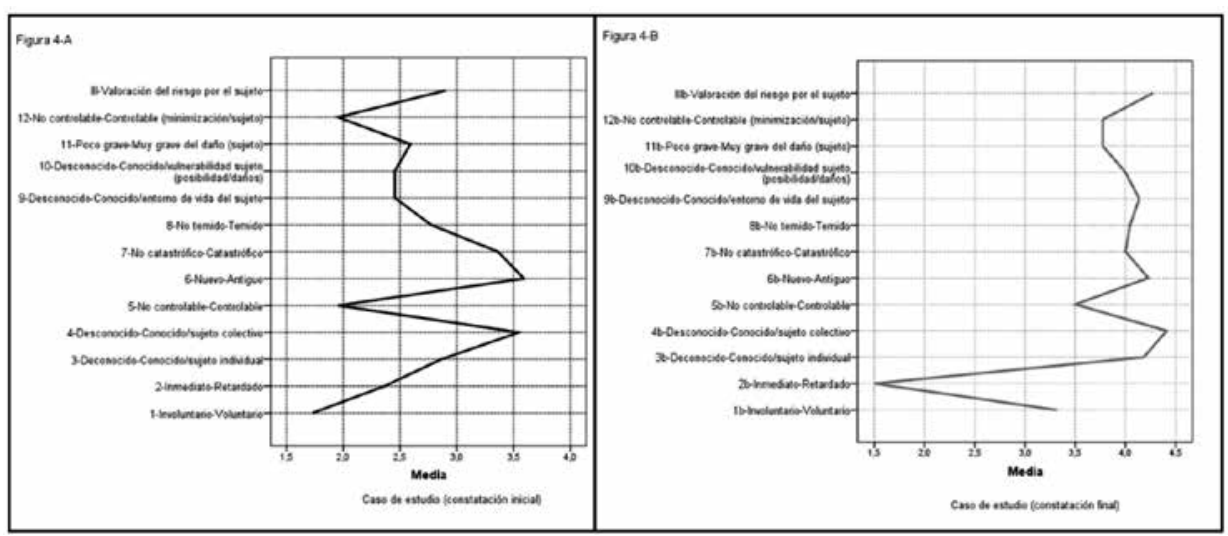

Fuente: Elaborado por el autor. 
Pablo Bayón Martínez. El pensamiento geográfico en la percepción de riesgos por peligros hidrometeorológicos extremos: estudio de caso Mariel, Cuba

DOI: http://dx.doi.org/10.15359/rgac.1-56.5

Estos perfiles corresponden con la sección II del instrumento de indagación (encuesta) en que se:

II-1: busca conocer la percepción sobre la voluntariedad o involuntariedad en la exposición al peligro por parte del sujeto, que articula con el reconocimiento de vulnerabilidades individuales y colectivas que repercuten en la gestión de riesgo. (Involuntariedad-voluntariedad ante el peligro)

II-2: explora la percepción sobre la demora de las consecuencias, parámetro crítico en el momento de explicar las actitudes y el comportamiento individual y colectivo del sujeto indagado. (Inmediatez del efecto)

II-3: explora el factor conocimiento que tiene el sujeto sobre el peligro. (Conocimiento del riesgo ante el peligro)

II-4: explora el factor conocimiento que el sujeto atribuye a los a los responsables de la prevención, en íntima relación con el conocimiento de los responsables, con la confianza en ellos y con la aceptación de las medidas preventivas que se proponen. (Conocimiento del riesgo por los actores-gestores institucionales y/o comunitarios)

II-5: indaga por el grado de control percibido, que permite descartar actitudes fatalistas recurrentes ("pasa cuando pasa y yo no lo puedo evitar"), o por el contrario sentimientos de excesiva confianza o de invulnerabilidad ("a mí no me sucederá esto porque soy más listo, ya he vivido otros y no es para tanto, tengo mayor experiencia, es más ruido que otra cosa, etc.") (Control-personal-sobre el riesgo)

II-6: explora la percepción del sujeto sobre la novedad o antigüedad del peligro, dado que la familiaridad con el peligro puede generar su no reconocimiento. (Novedad del riesgo con relación al peligro de estudio)

II-7: explora el potencial catastrófico que se atribuye al peligro, atributo que mantiene una relación alta y positiva con el riesgo total percibido y su gestión lo más racional y objetivo posible. (No catastróficoCatastrófico del peligro)

II-8: explora la respuesta emocional de temor, la característica más predictiva del peligro y del en general, percibido. (Temido o No temido).

II-9: explora el grado de conocimiento de la vulnerabilidad del entorno de vida del sujeto (condiciones naturales del lugar: zona baja, paleocauce, cercanía al mar, etc.; acueducto y alcantarillado; estado 
Pablo Bayón Martinez. The geographical thinking in the perception of risks for extreme hydrometeorological hazards: a case of study at Mariel, Cuba

DOI: http://dx.doi.org/10.15359/rgac.1-56.5

de la vivienda; etc.), ante el peligro respectivo. (Conocimiento del entorno y la vulnerabilidad)

II-10: evalúa la percepción del daño que tiene el sujeto individualmente, que articulada con la pregunta anterior, coadyuva a comprender el conocimiento de su vulnerabilidad o susceptibilidad ante el peligro, cuestión central en la gestión del riesgo. (Posibilidad que experimente el daño)

II-11: evalúa la percepción de la gravedad de las consecuencias, la que se corresponde con la magnitud de las pérdidas (de vida y/o materiales), que es una de las variables constitutivas de la definición técnica de riesgo, y se corresponde con la mayor o vulnerabilidad del sujeto ante el peligro respectivo. (Gravedad del daño)

II-12: trata de explorar tanto la visión que el sujeto tiene de su capacidad para realizar acciones preventivas (reducir la probabilidad de aparición del daño), como de realizar actuaciones para reducir el impacto del daño, lo que abunda en el grado de percepción de peligro por parte del sujeto. (Autogestión de riesgo del peligro).

La pregunta (sección III), es de tipo general y se dirige a obtener una estimación de la variable magnitud del riesgo percibido. La pregunta incorpora aclaraciones para estandarizar la gravedad de los desenlaces que se deben considerar (pérdidas de salud muy graves) y la latencia (tanto las consecuencias que suponen pérdidas de salud a corto plazo, como a medio o largo plazo).

La descripción general de los estadígrafos calculados y el perfil para cada atributo de percepción de peligros hidrometeorológicos y del riesgo global -por el sujeto- para cada momento del curso (inicial y final) respectivamente, revela que del peligro que identifica el sujeto como el que más le afecta individualmente o a su comunidad, los atributos relacionado con el "desconocimiento-conocimiento", "novedad-antigüedad" y la "valoración del riesgo", tienen valores de grado intermedio. Es importante significar que para el sujeto, la interacción con el peligro es principalmente "involuntaria" e "incontrolable" para la minimización de los daños a nivel individual y colectivo, lo que denota una actitud de vulnerabilidad cognoscitiva inicial.

Cada atributo fue calculado atendiendo a: sexo, grupo etario (jovenadulto-adulto mayor), nivel de instrucción vencido, situación laboral y tiempo de residencia en el territorio. 
Pablo Bayón Martínez. El pensamiento geográfico en la percepción de riesgos por peligros hidrometeorológicos extremos: estudio de caso Mariel, Cuba

DOI: http://dx.doi.org/10.15359/rgac.1-56.5

Los encuestados reconocen como principal peligro que les afecta al relacionado con las inundaciones por lluvia $(63,6 \%)$ lo que es contrario a la selección que registra la EPP de 2009-11, en que se reconoce al viento, coincidente con el criterio de los especialistas, como el fenómeno de mayor peligrosidad histórica para la región, cuestión que es percibida por los sujetos a través del proceso educativo desarrollado, como parte de la formación de bases cognoscitivas acerca de los peligros hidrometeorológicos en Cuba en general y en el municipio Mariel.

En la constatación realizada al culminar el curso, se percibe mayor nivel de concientización y conocimientos acerca de los peligros hidrometeorológicos extremos, relacionados en primer lugar con el viento, que afectan al sujeto en mayor o menor medida según las particularidades y contexto relativo, siento las inundaciones por intensas lluvias o las penetraciones del mar, la que ocupan en lugar principal en las percepciones individuales.

Como se observa en la figura 4-B se muestra un incremento relativo en el grado de percepción del sujeto para cada atributo del riesgo de peligro analizado -en grado alto y muy alto- y para todas las variables de estudio, al finalizar el curso de capacitación concebido, lo que avala el planteamiento teórico (objetivo general) inicial, acerca de la contribución del conocimiento geográfico (educación geográfica) a la percepción (y gestión) de los riesgos de desastres por peligros naturales, en los espacios/ territorios de asimilación-reproducción de la vida social en el contexto del desarrollo local.

\section{Resultados: reflexiones conclusivas}

Se constata empíricamente (constatación inicial) la tendencia generalizada del sujeto de percibir en grado intermedio a bajo y muy bajo, y por tanto, a la deficiente percepción del riesgo del peligro valorado, para los diferentes ámbitos (variables) de análisis, donde se exploran las características siguientes:

- Deficiente reconocimiento de las vulnerabilidades individuales y/o colectivas que potencian el riesgo respectivo.

- Pobre consideración al potencial destructivo y consecuencias derivado del fenómeno, con independencia a la relativa confianza en las autoridades e instituciones comunitarias. 
Pablo Bayón Martinez. The geographical thinking in the perception of risks for

extreme hydrometeorological hazards: a case of study at Mariel, Cuba

DOI: http://dx.doi.org/10.15359/rgac.1-56.5

- Se registran deficiencias cognoscitivas acerca del fenómeno hidrometeorológicos, sus efectos y maneras de minimizar sus efectos cuando se producen (no controlable-controlable). En sentido general existe predisposición acerca del carácter impredecible e inevitable del fenómeno y sus consecuencias, al margen de cualquier control o minimización por parte del sujeto (social).

- En contradicción con lo anterior, el grado de "temor" es relativamente bajo; quizás condicionado por el contacto histórico con este fenómeno y la relación directa con la alta atención y gestión estatal (institucional, comunitaria, etc.), de protección civil.

- La población adulta (30-59 años), manifiestan mayor grado de temor inicial cercano al grado "intermedio".

- $\quad$ Otro aspecto manifiesto, se centra en el grado de familiaridad del individuo con el peligro (II-6; novedad o antigüedad), con la tendencia la minimización del temor por los mismos, tendencia predictiva más general, propiciadora de conductas disímiles ante el fenómeno peligroso de estudio.

- Contrario al elemento anterior, es de alto reconocimiento, el nivel del daño (II-7; no catastrófico-catastrófico) del fenómeno, percepción mayormente diferenciable -en sentido positivo- de la población jubilada, y de mayor tiempo de residencia en el territorio.

- $\quad$ En sentido general, se revela el insuficiente conocimiento -por parte del sujeto- de su entorno de vida cotidiana, de las vulnerabilidades sociales y geográficas que le circundan (II-9 y II-10), con excepción de los jubilados y los residentes de 10 años o más en el territorio, precognición dada por experiencia vivida. El resultado inicial no es proporcional con el nivel de instrucción del sujeto, que por demás es alto (Media Superior y Superior), que ha criterio del autor de la presente investigación, manifiesta insuficiencias educativas en todos los ámbitos (formal, informal, no formal), con independencia de la voluntad política y esfuerzo institucional y de los demás actoresgestores del proyecto social socialista cubano, con más de 56 años de experiencia.

- $\quad$ Para casi todos los encuestados, con independencia de las variables sociodemográficas estudiadas, ven reducida su capacidad para realizar acciones preventivas para reducir la probabilidad de aparición 
del daño (II-12; autogestión de riesgo del peligro), lo que muestra baja percepción, y al cambio de actitud (social) de enfrentamiento y superación del riesgo como actor individual y colectivo, en el escenario de vida respectivamente.

- Los perfiles resultantes de la constatación empírica final, muestran resultados superiores al intermedio y alto, y en algunos casos muy alto, para casi todos los atributos de riesgo, en sentido favorable, con relación a la mayor percepción del riesgo por parte del individuo.

- $\quad$ Estos resultados reafirman el planteamiento teórico propuesto acerca del impacto de la educación geográfica en la percepción de riesgo por peligros naturales, dado por la contribución del conocimiento de las condiciones geográficas de los espacios de vida cotidiana del sujeto a la formación cultural de la población, en el manejo integrado de sus riesgos previo la identificación de sus vulnerabilidades.

\section{Conclusiones}

La percepción -como acto de naturaleza cognitiva- es estudiada desde diversos puntos de vistas, siendo amplia y diversa las investigaciones aplicadas y de autores, que refieren al respecto, con miradas desde las perspectivas sicológica y sociológica, aplicada en diferentes ámbitos de la vida social, que a los intereses de la presente investigación, se suscribe a la dimensión de la percepción de riesgo, por peligro natural, desde la interacción compleja entre las categorías de espacio natural, espacio económico y de hábitat, espacio social y espacio cultural, de los sujetos sociales (individual y colectivo), en torno a los peligros naturales contextualmente.

Se aporta a la gestión de riesgo de desastre de los territorios, en general, y al caso de estudio, en particular, de las premisas geográficas para la percepción ambiental respectiva, como parte de la construcción social cotidiana, contribuyendo a la materialización de las misiones institucionales, y personales, en relación con el manejo de riesgo de desastres en Cuba, el cambio climático, entre otras prioridades de este carácter. Acompañado de una propuesta de gestión educativa conforme a los escenarios de riesgos y actores involucrados, como parte del manejo integrado de riesgos, en el contexto de los planes del Centro de Gestión de Riesgo local. 
Pablo Bayón Martinez. The geographical thinking in the perception of risks for

extreme hydrometeorological hazards: a case of study at Mariel, Cuba

DOI: http://dx.doi.org/10.15359/rgac.1-56.5

\section{Referencias}

Almaguer, C. (2008). El riesgo de desastres: una reflexión filosófica. Tesis doctoral. Ministerio de Educación Superior, Universidad de La Habana Facultad de Filosofía, Departamento de Filosofía. Cuba. p.162.

Bayón, P. (2014). Cultura ambiental: educación y ética para su formación. En: Cooperativismo, producción y reproducción de la vida y cultura ambiental. Cuba: Editorial Caminos.

Consejo de la Administración Provincial, Artemisa. (2012). Estudio de peligro, vulnerabilidad y riesgos por fuertes vientos, penetraciones del mar e intensas lluvias. (Informe provincial). La Habana.

Castañeda, G. (2014). Percepción del riesgo a huracanes en cinco comunidades costeras del estado de Colima. En: Basil Rodríguez, A.; Contreras Montellano (coordinadores): La construcción del futuro: retos de las ciencias sociales en México. Memorias del 4to Congreso Nacional de ciencias Sociales; IV Sociedad y ambiente: vulnerabilidades, riesgos y exclusión. pp. 691-702. Publicado por CESMECAUNICAHC, Tuxtla Gutiérrez y COMECSO, Tijuana.

Conesa, C., Calvo, F. (2003). Introducción: Los procesos de riesgo con origen natural: una constante en la relación entre hombre y medio". En Revista de Ciencias Sociales, 23. Murcia. Universidad de Murcia. Recuperado de http://digitum.um.es/xmlui/bitstream/10201/20141/1/ Introducci\%C3\%B3n.\%20Los $\% 20$ procesos $\% 20 \mathrm{de} \% 20$ riesgo $\% 20$ con\%20origen\%20natural.pdf

Delgado, C. (2005). Efectos del desarrollo científico-técnico: sensibilidad pública, conocimiento y riesgo. En: Valdes Menocal, C. (comp.). Ecología y Sociedad. Selección de Lecturas. La Habana: Editorial Félix Varela, 2005. pp. 223-240)

Espluga, J. (2002). Las dimensiones psicológicas, sociales y culturales del riesgo y su relación con la prevención de riesgos laborales. Ponencia presentada en la Jornada Técnica: La nueva cultura de la prevención: de la culpabilidad al compromiso. Barcelona, Recuperado de http://www.mtas.es/insht/research/JEspluga.htm

Fischoff, E., Slovic, P. y Lichtenstein, M. (1979). Which Risks Are Acceptable? Environment, 21(4) p.13

GNER-AMA. (2010). Lineamientos metodológicos para la realización de los estudios de Peligro, Vulnerabilidad y Riesgos de Desastres 
Pablo Bayón Martínez. El pensamiento geográfico en la percepción de riesgos por peligros hidrometeorológicos extremos: estudio de caso Mariel, Cuba

DOI: http://dx.doi.org/10.15359/rgac.1-56.5

de inundación por penetraciones del mar, inundaciones por intensas lluvias y afectación por fuertes vientos. [Grupo Nacional de Evaluación de Riesgos- Agencia de Medio Ambiente]. Ministerio de Ciencia, Tecnología y Medio Ambiente. (Serrano, Herminia -et al-). (Documento de actualización y reajuste). [Resultado de "Implementación de los estudios de PVR para la reducción de desastres para la República de Cuba". Estado Mayor Nacional de la Defensa Civil, 2006].

GNER-AMA. (2013). Base de Datos Nacional, de percepción de riesgo por peligros hidrometeorológicos. Grupo de PVR-AMA. [Elaborado por: Cristina López-Calleja Hiort-Lorenzen, Lilia Núñez Moreno, Elizabeth Godefoy Núñez.

Lobato, R. (1995). Um conceito chave da Geografia., In: Geografia: conceitos e temas., Rio de Janeiro, Bertrand Brasil, pp. 25-48.

Marx, C. (1974). Tesis sobre Feuerbach. En: Marx, C.; F. Engels. Obras Escogidas. Moscú: Editorial Progreso. p. 24-26

ONEI. (2010). Artemisa. Oficina Nacional de Estadísticas e Información. Cuba. Recuperado de http://www.one.cu/publicaciones/cepde/ Nueva\%20DPA/NUEVA_2010_ARTEMISA.pdf

ONEI. (2012). Anuario Estadístico de Cuba, Edición 2013. Consultado: 2005-2014. Recuperado de http://www.one.cu/aec2012/datos/00\%20 ANUARIO\%20ESTADISTICO\%20DE\%20CUBA\%202012.pdf

Puy, A. (1994). Percepción social del riesgo. Dimensiones de predicción y evaluación. Tesis presentada en opción al grado científico de Doctor. Universidad Complutense de Madrid. Facultad de Psicología, Departamento de Psicología Social. 469 p.

Puy, A. y Aragonés, J. (2003) Percepción social de los riesgos y gestión de las emergencias ambientales Desastres y Sociedad. Red de Estudios Sociales en Prevención de Desastres en América Latina, 8(5). Recuperado de http://www.desenredando.org 39

Slovic, P. y Weber, E. (2002). Perception of Risk Posed by Extreme Events. Decision Research and University of Oregon Columbia University and Wissenschaftskolleg zu Berlin. Conference Risk Management strategies in an Uncertain World.. Palisades, New York, April 12-13, 2002. Recuperado de http://www.ldeo.columbia.edu/chrr/ documents/meetings/roundtable/white_papers/slovic_wp.pdf 